\title{
Toolset for evaluating infrared countermeasures and signature reduction for ships
}

\author{
Ric (H.)M.A. Schleijpen. \\ TNO Defence, Security and Safety, P.O. Box 96864, \\ 2509 JG The Hague, The Netherlands,
}

\begin{abstract}
The protection of ships against infrared guided missiles is a concern for modern naval forces. The vulnerability of ships can be reduced by applying countermeasures such as infrared decoys and infrared signature reduction.

This paper presents a set of simulation tools which can be used for assessing the effectiveness of these measures. The toolset consists of a chain of models which calculate the infrared signature of a ship (EOSM), generate an infrared image of the ship in a realistic sea and sky background (EOSTAR) and determine the behaviour of an infrared missile seeker against these images and simulate the complete missile fly-out including countermeasure deployment (EWM). All model components will be discussed. Typical simulation results will be shown.
\end{abstract}

Keywords: Infrared signatures, Infrared countermeasures, Anti Ship Missile Defence

\section{INTRODUCTION}

The operational effectiveness of a navy ship depends on the ability of a ship to perform its tasks in a conflict situation. One important aspect of the operational effectiveness is the survivability. The survivability is determined by:

- Susceptibility: the process of the detection of a platform up to the probability that the platform will be hit by an enemy weapon.

- Vulnerability: the level of reduction of operational capabilities of the platform after a hit.

- Sustainability: the level of availability of the essential systems on board of a platform during a full operation.

At a sublevel of the susceptibility the key element is to avoid being hit by missiles. Against infrared guided anti ship missiles, the combination of infrared ship signature reduction and the deployment of infrared decoys can be very effective in hit avoidance. Various techniques for signature reduction have been developed over time. For example thee effect of exhaust gas cooling by either mixing cool air or water with the exhaust gases have been studied in reference 1 and 2 respectively. Cooling the hull and parts of the superstructure with cold water from the pre-wetting installation on board of the ship is another way of reducing the signature which has been presented in reference 3 . Infrared decoys can be used to interrupt the guidance from a missile towards the ship. The timing and the direction of the decoy launches is very critical for the success and many studies have been dealing with this issue, as for example reported in references 4 and 5.

At a sublevel of the vulnerability the actual point of impact or "hit point" in a missile attack is a key element. Current studies aim for a better understanding of the aim point selection in missile seekers. The ideal situation would be to use this understanding to adapt the ship design in order to avoid being hit in critical spots on board of the ship [6].

A need for methods for quantitative analysis of the effectiveness of the countermeasures is what all the efforts to reduce the susceptibility and vulnerability have in common. Reference 1 describes a method where the lock-on range is used as a figure of merit. A set of generic missile seekers ranging from very basic hot spot seekers to advanced imaging seekers has been defined. The effect of signature reduction is expressed as a reduction in lock-on ranges. This is a simple approach, which does not need very complex simulations, allowing a rapid comparison between the signatures of various

ric.schleijpen@tno.nl, phone +31 70374 0045, fax +31 70374 0654, www.tno.nl

Technologies for Optical Countermeasures VII, edited by David H. Titterton, Mark A. Richardson, Proc. of SPIE Vol. 7836, 78360F · @ 2010 SPIE · CCC code: 0277-786X/10/\$18 · doi: 10.1117/12.869849 
ship configurations. The disadvantage is that the lock-on range cannot be converted directly into a figure of merit for the susceptibility. Despite reduction in lock-on range the missile will eventually find and hit the ship, if the ship is in the search region of the missile and no countermeasures are deployed. This indicates the need for a tool to measure the effect infrared signature reduction on the survivability more directly than the decrease in lock-on range.

References 4 and 5 describe the use of recorded infrared image sequences of ships launching decoys for the determining the effectiveness of the decoys. In this case the final decision of the seeker to track either the ship or the decoy is the figure of merit. The advantage of this approach is that it results in a figure of merit which relates directly to the susceptibility of a ship. Various seeker tracking algorithms can be applied on the image sequences, simulating a range of missiles which results in a more generic result than just one seeker. However, the use of recorded images limits the evaluation to the conditions for which the images were recorded. A full evaluation would require more missile approach angles and different decoy deployments. These examples of evaluation efforts explain the need for a method which can evaluate signature reduction and infrared countermeasures under many different conditions and scenarios and which provides results which can be converted directly in a figure of merit relevant for the survivability.

This paper will present a set of simulation tools which can be used for assessing the effectiveness of countermeasures measures, based on closed loop simulations of infrared missile engagements against ships.

The set-up of the toolsets will be discussed in section 2. Section 3 and 4 will show results from simulations for studies on countermeasure effectiveness and hit point analysis respectively. These studies use two subsequent generations of the tool set. Summarised findings and conclusive remarks are given in Section 5.

\section{MODEL COMPONENTS: TOOLSET}

The toolset consists of a chain of models which:

- calculate the infrared signature of a ship based on the ship design,

- generate an infrared image of the ship combined with sea foreground and sky background and

- determine the behaviour of an infrared missile seeker against these images and simulate the complete missile fly-out including countermeasure deployment.

Figure 1 shows schematically the relation between the components of the toolset.

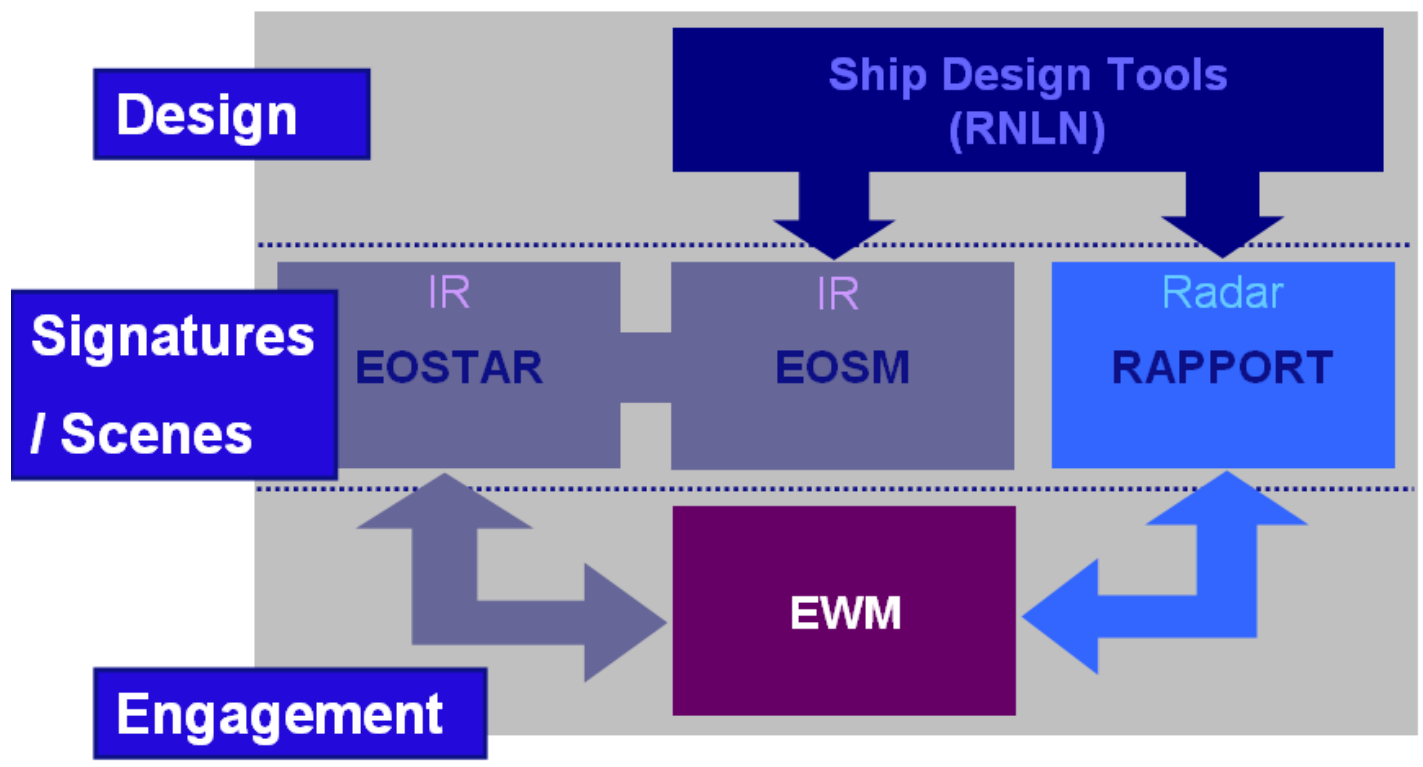

Figure 1: relation between the components of the toolset 
The common part in the simulations described in sections 3 and 4 is the engagement part of the model. This part is based on the Electronic Warfare Model (EWM). EWM is a system that is capable of performing closed-loop simulations regarding the engagement of missiles against ships. It is a TNO in-house development which was originally designed for simulation of radar guided missile engagements. EWM now includes detailed seeker models both for infrared and radar guided missiles. These seeker models simulate the details of the interaction of the seeker with targets and decoys. The model simulates the decoy deployment, for both RF (Chaff) and Infrared decoys. This includes the timing of the decoy launches, the calculation of the position where the decoys bloom and the subsequent drift of the decoys by the wind. Since the decoy effectiveness depends to a large extent on the location of the decoys with respect to the ship (horizontal distance and altitude), this is a critical factor in the simulations. The missile flight dynamics are also simulated in a detailed manner, including a 6 degree of freedom missile fly-out model. The targets can be represented in different levels of detail, depending on the requirements in the study. For the RCS (Radar Cross Section) the signature is represented by a number of scatter centres. In the minimum configuration six scatter centres are located at symmetric positions and are equal in strength. In the more advanced approach, the location and strength of the scatter centres are derived from detailed RCS models of the target. Statistical variations are applied on the scatter centre to simulate the glint. The level of detail of the representation of the target in the infrared also depends on the requirement for the study and will be described for two particular cases in sections 3 and 4 of this paper. A more detailed description of EWM is given in reference 7 .

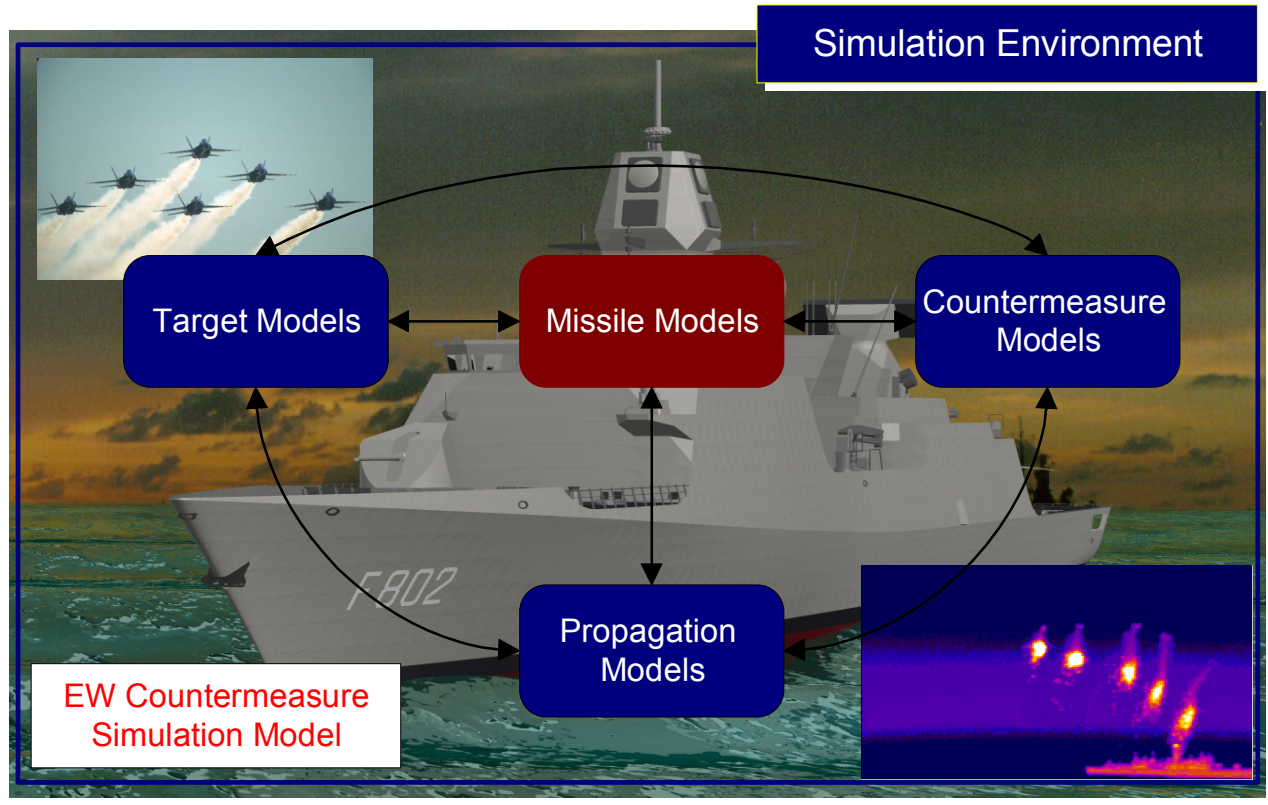

Figure 2: Schematic overview of the TNO Electronic Warfare Model

The simulation calculates the trajectory of the threat missile during the engagement. When this trajectory passes through a box representing the size of the ship, the engagement is defined as a hit. When the missile misses the target the miss distance is calculated. A typical analysis involves a sequence of simulations over different attack directions, different ship velocities and wind speeds and wind directions. For each combination 10 repeat runs are calculated to achieve a statistical average. This results in a total of a few thousand runs per configuration. Tools are available to process, analyse and summarise these results. Individual engagements can be visualised to check. The visualisation tools show the complete trajectory of the missile and the deployment of the decoys. Also details such as the seeker look angle and the status of the seeker logic are stored for each step in the simulation to support the interpretation of the results.

The EW model has been used to develop decoy countermeasure deployment strategies, including combinations of decoys and jamming. The deployment strategies have been implemented in a Tactical Decision Aid (TDA) for optimum countermeasure deployment, currently in use on board of platforms of the Royal Netherlands Navy. The TDA calculates the optimum timing of the decoy launches and provides advice on ship manoeuvres in order to generate the maximum 
miss distance. A derivative of this TDA is also implemented in the EW model to automatically optimise the decoy deployment in the simulations; alternatively the decoy deployment can be programmed manually for the simulations.

\section{CASE 1: INFRARED DECOY EFFECTIVENESS}

Two recent Infrared decoy effectiveness studies have used the toolset. The goal of the first study was to develop and test a Tactical Decision Aid (TDA) for optimum infrared decoy launch strategy. The goal of the second study was to advise the on the optimum type and configuration of decoy launchers for new platforms of the Royal Netherlands Navy. The focus of both studies was to determine whether a missile seeker would continue to track the ship or a lock-transfer to the decoy would occur. The missile seeker takes this decision at relatively large distances from the target. Therefore, less detail in the target representation is required. In this case the target is built form 3D blocks with a uniform temperature. Typically 3 to 4 blocks are used to represent the hull, the superstructure, the stack and the exhaust gas plume. The target is positioned in a sea-sky background. The sea and sky background are uniform in temperature with a noise level superimposed. No waves and clouds are simulated, thus creating the most difficult case for the decoys to achieve success against the missile seeker. Since the anti ship missiles only search near the horizon, no efforts were made to simulate the vertical gradients in sea and sky radiance which occur in real infrared scenes. The decoys are simulated by hot areas in the scene. As can be seen from the examples in figures 3 and 4, the decoys can in general easily compete with the ship signature in intensity. In fact the most critical factor in the effectiveness is found to be the position of the decoy with respect to the target as seen by the seeker. The actual position and drift of the decoy is calculated in detail by the EW model. Basically this reduces the decoy effectiveness to a geometrical problem, which justifies the simplified approach in target and background representation. Using this simplified approach allows running a few thousand simulations on a PC in a few hours.

Two seekers are used in this simulation: a hot spot seekers and an imaging seeker. The design of the imaging seeker is inspired by a linear array detector, scanning the horizon. For target detection a threshold level is derived for each line of the image based on the noise level near the edges of the image. The area above or below the threshold is marked as the target. These areas from the different lines are combined to one target area and a track box is defined around the target, leaving a small margin around the target. In the acquisition phase the seeker scans the horizon from left to right and vice versa. When the target is detected in two subsequent scans the track-box is initiated and the centre of the track-box is taken to derive the tracking point. During the tracking phase, only the area within the tracking box is evaluated. The size of the track box increases when the size of the area with intensity above the detection threshold increases. This is done to accommodate the apparent growth in size of the ship target when the missile approaches the ship, but it also occurs when a decoy drifts into the tracking box. In case there are two potential targets in the track box, the seeker selects the largest target as the true target. In principle the tracker counter-counter measures could be enhanced by evaluation target features such as the average grey level and the ratio between horizontal and vertical size of the target. These features will help to discriminate between decoy and ship when decoys have entered the track box. This feature analysis was not implemented in this version, because in the typical conditions used in the study it turned out that getting the decoy in or near the track box was difficult enough.

Figure 3 shows an example of typical decoy sequence, where no lock-transfer was achieved. The decoys were launched and ignited at an altitude above the track box. Although the decoy clouds descended and just entered at the edge of the track box the seeker decided to continue to track the ship, because the seeker selected the larger ship as the true target. Figure 4 shows the result of an alternative decoy deployment. The decoys are smaller and are fired closer to the line of sight from the seeker to the ship. Also the distances between the decoys are smaller, which results in a single decoy cloud which is seen by the seeker as a large target. In this case the seeker transfers the lock-on to the decoy. 


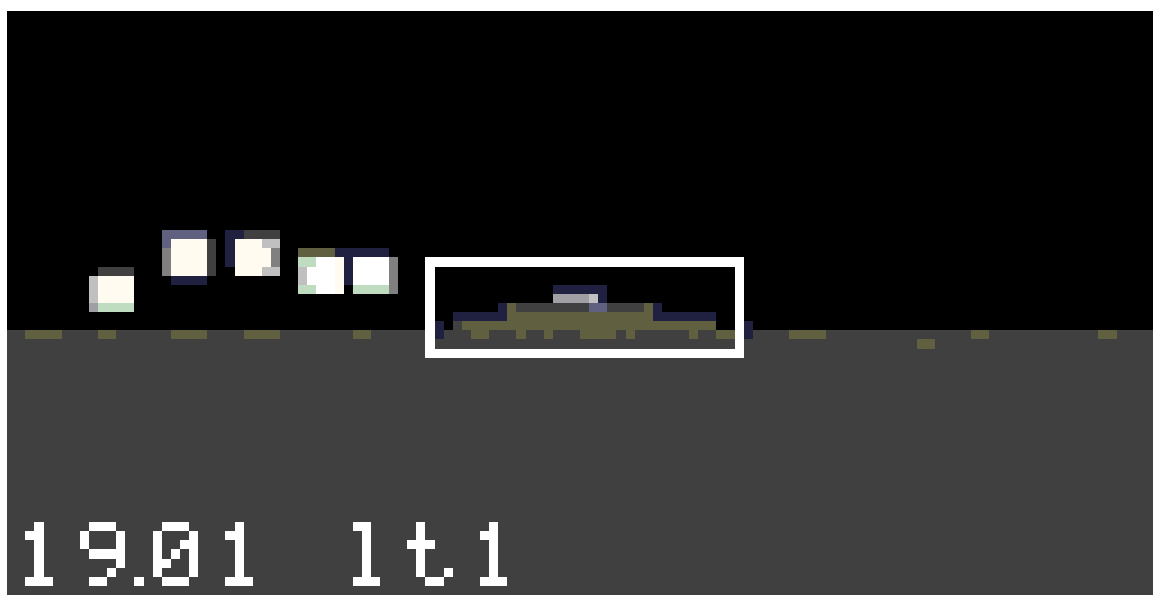

Figure 3:Standard decoy deployment against an imaging IR seeker

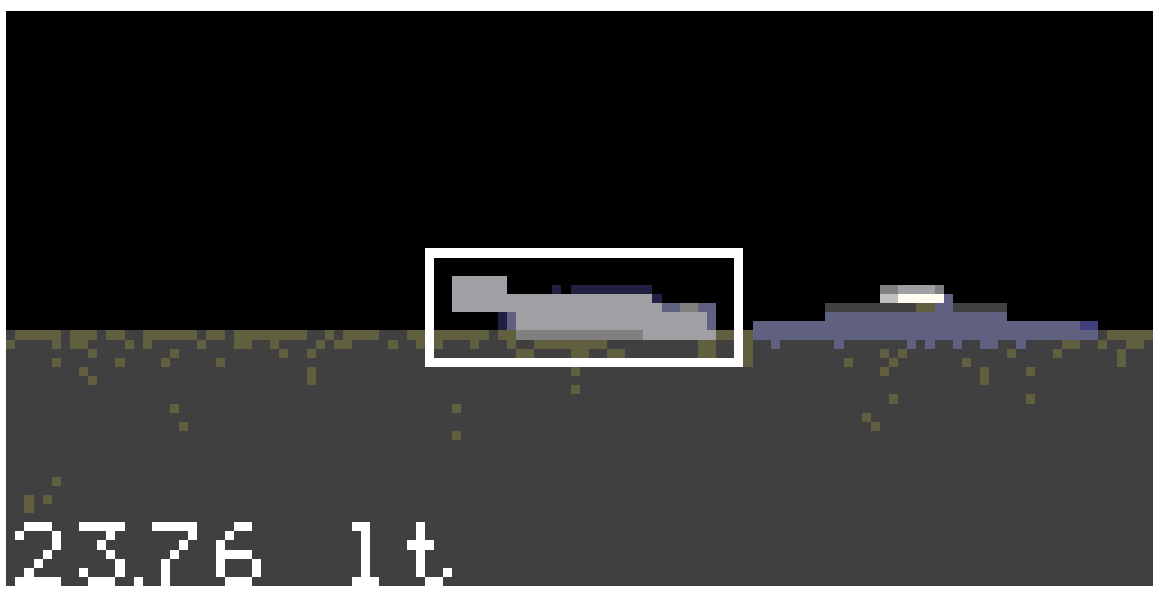

Figure 4: Advanced decoy deployment against an imaging IR seeker

\section{CASE 2: HIT POINT ANALYSIS}

The hit point analysis studies have been performed in relation to the question: How to design a ship, that will not be hit by anti-ship missiles in certain parts of the ship, for example the command centre, the ammunition stores or the engine room. The next step is to develop simulation models to support the design phase and use these models to test new designs. The seeker takes its decision on the hit point at a much smaller range compared to the situation in case 1 for the decoy effectiveness studies. Therefore, there is a need for a more detailed representation of infrared ship imagery. For this application the infrared image sequences are generated with the EOSTAR model. EOSTAR is a flexible tool to assess the performance of electro-optical sensors in the maritime environment (reference 8). This includes detection range calculations against specific targets. EOSTAR provides a toolbox with modules to evaluate the main atmospheric processes, such as transmission, refraction and turbulence. Background and signature models allow the generation of synthetic camera images for a scenario of interest. The thermal radiance properties of the scene elements in these images are calculated in detail. For example the sea foreground model ensure that the images have wave shapes and spatial frequencies corresponding to measured data. A sensor module, including optical blur and signal processing algorithms, generates the synthetic image as seen by an operator or the sensor. These synthetic images consist of a ship placed in a sea foreground and against a sky background with clouds. The actual infrared signature is generated with the EOSM (Electro-Optical Signature Model) module of EOSTAR. EOSM is a tool for calculating infrared signatures of military platforms by solving detailed heat balance equations. In an international comparison of infrared ship signature prediction 
models based on data from a NATO trial, EOSM performed very well and comparable to the SHIP-IR model (reference 9). See Fig. 5 for an example of an infrared signature of the M-frigate of the Royal Netherlands Navy that was generated with EOSM. The M-frigate data were used to test the simulation sequence, because both ship design and engineering data and numerous measurement data were available, allowing good comparison.
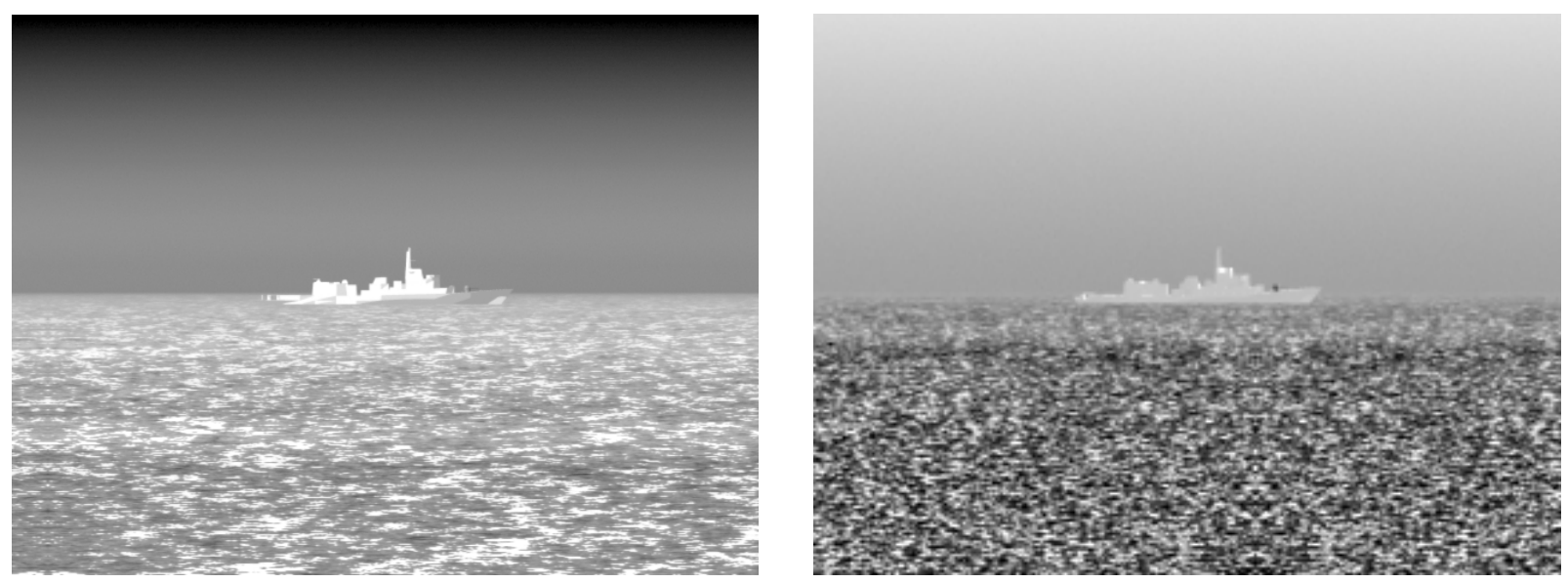

Figure 5 Typical images generated with the EOSTAR EOSM tools. Left panel: LWIR. Right panel: MWIR.

Four different infrared imaging seeker models were implemented (Table 1). It is expected that imaging seekers will have the best capabilities to select a specific aim point on the target. The current implementations of the imaging seeker models are the best available at this time.

Table 1. Infrared seekers used in the hit point analysis.

\begin{tabular}{|l|l|l|}
\hline Seeker 1 & Imaging: Correlation tracking & Open loop simulation \\
\hline Seeker 2 & Imaging: Centroid tracking & Open loop simulation \\
\hline Seeker 3 & Imaging: Track gate & Closed loop simulation with EWM \\
\hline Seeker 4 & Hot spot & Closed loop simulation with EWM \\
\hline
\end{tabular}

The various seeker models allow gaining a good understanding of the effects of the various tracking algorithms on the aim point selected by the seeker. It has to be noted that the image processing capability of imaging seekers should be able to select a specific part or section of the ship as the aim point. However, most often this type of processing requires quite some a priori knowledge like the aspect angle under which the approach will take place. This has consequences for the robustness of the tracking algorithm. Therefore, we assume that this type of processing will possibly be implemented in future seekers, but is not yet implemented in the current generation of seekers.

All seekers used in the hit point analysis, have been developed in the past for other simulations. For none of the seekers a special final homing algorithm was implemented. Seeker models 1 and 2 represent seekers with more advanced image processing than the imaging seeker 3. Seeker 3 is essentially the same seeker as used in the countermeasure effectiveness study. The hotspot seeker was used as a reference. Both seekers 3 and 4 are described in reference 7. Seeker models 1 and 2 were developed to be used in combination with high quality image sequences of tanks in a land background, while seeker models 3 and 4 were developed for use in the EWM in combination with a very rough representation of the ship. None of the seekers was specifically tuned for the ship image sequences. A good tuning of the seekers to the quality of the new image sequences requires quite some effort, to arrive at a seeker algorithm which is robust over a wide range of circumstances covering low contrast and high contrast targets, targets with and without countermeasure deployment and all aspect angles.

It was decided to limit the number of scenarios to two weather conditions, clear sky and full overcast at typical midlatitude atmospheric conditions. Full overcast gives a condition where the surface temperature of the ship is mainly 
determined by the ambient air temperature. In the clear sky condition the solar heating of the ship has to be taken into account. Maximum and minimum contrast is than expected for the sun and shadow side of the ship respectively. The sky background and the sea foreground models in EOSTAR are derived from models for IRST (Infrared Search and Track) system evaluation. Because an IRST system on board generally does not move very fast over the water surface, the effects of fast movement over the sea surface are not included in the foreground model. This makes the foreground in the image sequences look somewhat unrealistic. However, this does not affect the seeker behaviour. Therefore, we considered it not necessary to improve this part of the scene generation for the hit point analysis.

The intensity of the plume from the smokestack has a strong effect on the ship signature especially in the MWIR (3$5 \mu \mathrm{m})$ band. For this band, infrared images for three different power settings were calculated resulting in three different plume conditions, i.e. a large plume from a gas turbine engine, a medium size plume for a diesel engine and no plume at all. In the LWIR band the effect of the plume is much less dominant and therefore the LWIR images are calculated without plume. These combinations of weather conditions and plumes result in twelve scenarios.

For the hit point simulations the image sequences were derived by converting the radiance map directly to grey levels in the images. In reality the optics and the detector sensitivity of the camera or the missile seeker have to be included as well. Currently this can be done as a part of EOSTAR as well. The new approach may result in some differences in the grey levels, but this will not influence the main conclusions for the hit point analysis.

The seekers 1 and 2 were used in an open loop simulation, where an image sequence was generated in advance with the target always positioned in the centre of the field of view. The anti-ship seekers on the other hand were used as a part of the EWM model that allows for closed loop simulations. Based on the seeker tracking output, EWM calculates the new line of sight direction for the missile seeker and provides this as an input for EOSTAR.

The simulations with seeker 1 and 2 were done for all 12 scenarios; for seekers 3 and 4 only a subset has been used, not using the overcast sequences. All simulations are done for broad side (port side or starboard side) approach of the seeker to the ship. For this paper a selection of the results for the open loop simulations with the seekers 1 and 2 is shown in figure. $6 \mathrm{a}-6 \mathrm{~d}$. The figures show the final image in each simulation sequence where a purple marker indicates the aim point selected by the seeker. The green marker indicates the centre of the field of view.

6a.

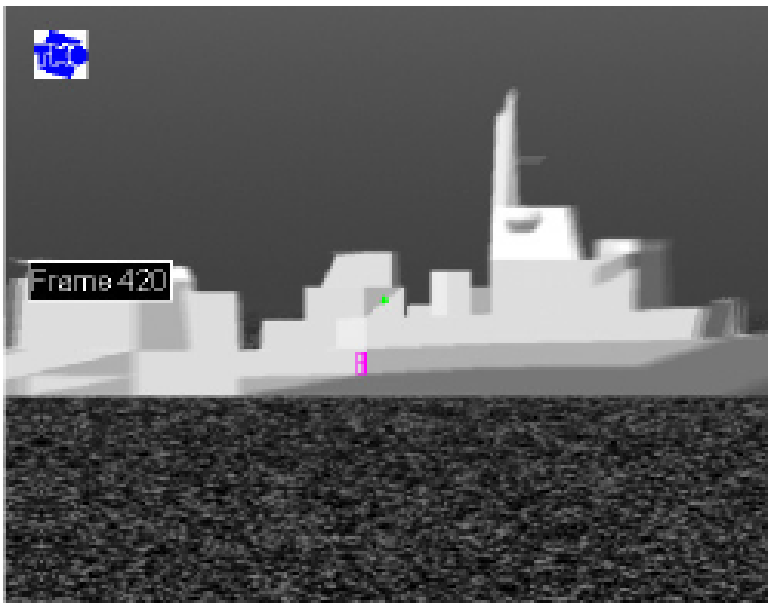

$6 \mathrm{~b}$.

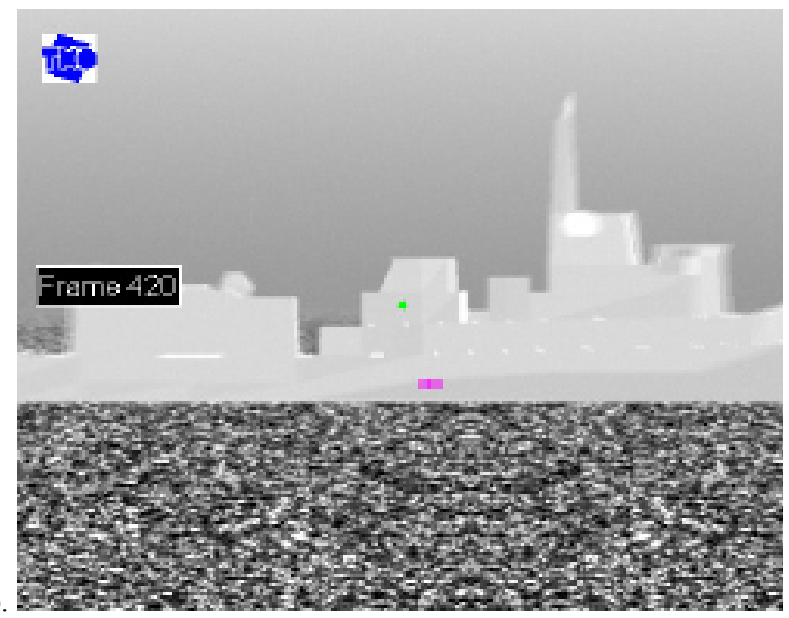

Figure 6. a) Stable track. Final aim point on the hull. b) Less stable track, due to the lower contrast on the shadow side. 

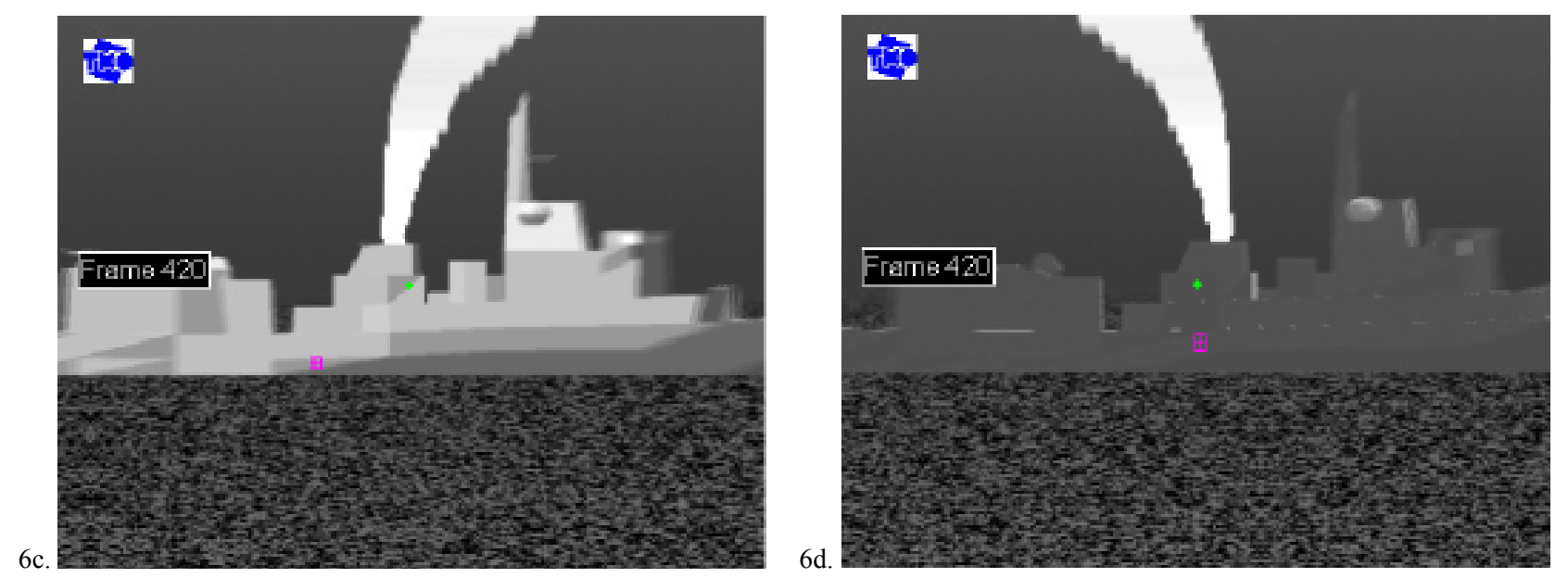

Figure 6. c) Plume seems to be ignored by the correlation tracker. Similar result as in scenario 1. d) Plume seems to be ignored by the tracker. The hot plume has effect on the dynamic range, which compresses the contrast on the ship itself. Note that the wind direction has reversed.

A selection of results for the simulations with seeker 3 is shown in Figures $7 \mathrm{a}-7 \mathrm{~d}$. As for seekers 1 and 2, the seeker parameters have not been optimised for these simulations. The size of the hot spot detector area was simple copied from previous simulations which might have had a different spatial resolution in the image, resulting in a different instantaneous field of view for the seeker. This then may influence the size of the hot spots as "seen" by the missile seeker. Despite the fact that the seeker parameters have not been tuned for these specific image sequences, the settings used are still considered to be representative for a realistic seeker.

For each scenario typically 10 runs were performed. The difference between the runs is caused by the noise which is added to the pointing commands for the seeker gimbal. Effectively this introduces variations on the centre position of the image. For each scenario the horizontal position of the hit points of the ten simulation runs are indicated by a yellow bar against a generic background picture of the ship, in the left part of the figure. This background image is for visualisation only and clarifies the dimensions of the hit point in reference to the ship. The right hand part of each image shows the typical hit point for a single selected run with enlarged pictures showing the detailed aim point. In the pictures a yellow vertical line represents the centre of the ship from a lateral point of view. The vertical dashed white line indicates the average hit point. Its uncertainty is marked by two short vertical red lines. Extra hit point sections are marked by vertical dotted lines. The spread for such sections are marked by a different colour. The small open red square is a hit point for one selected run. 

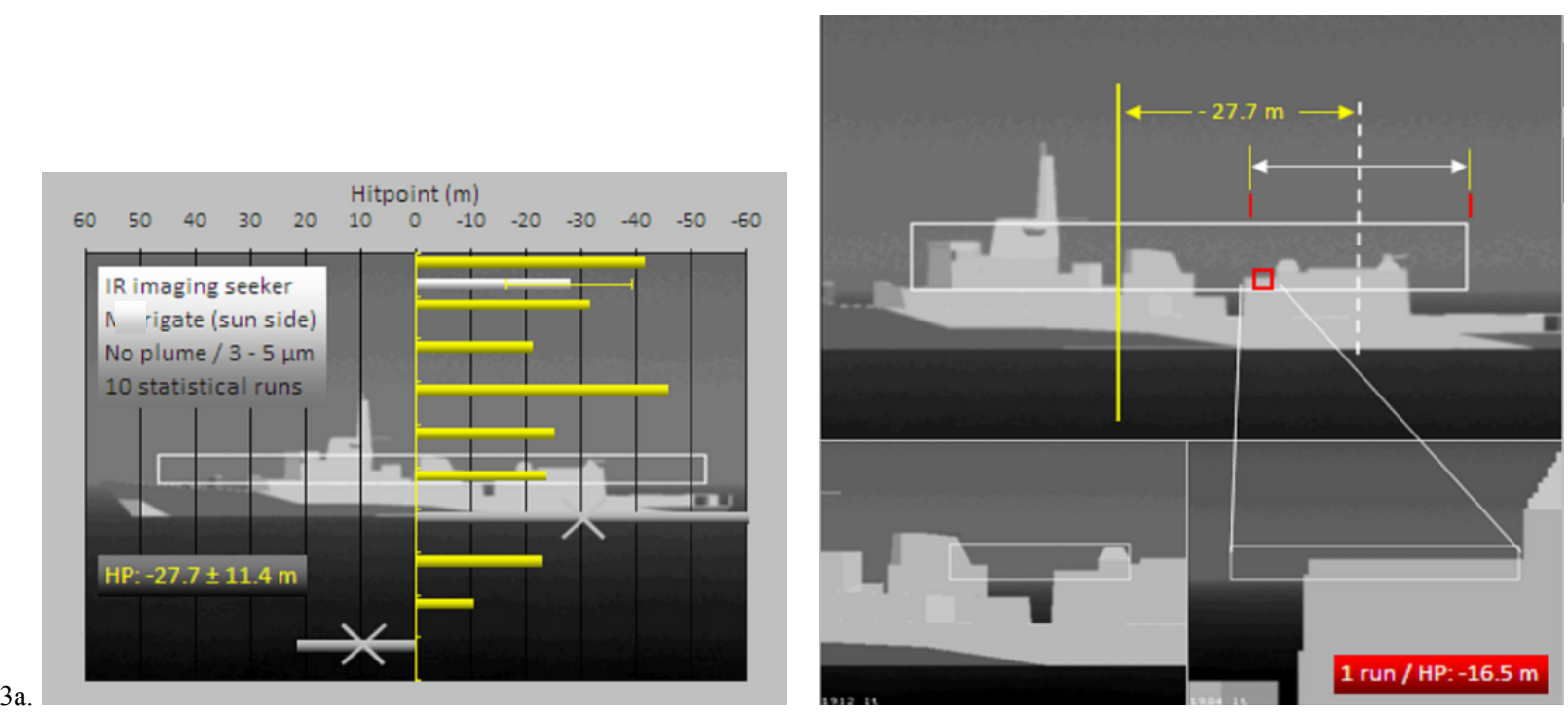

Figure 7a. Hit point results for scenario 1. Eight successful runs show the average hit point in the back of the ship at approx. -28 meter from the centre (left panel. Two outliers have been left out. Right hand panel: a single run hit point - the open red square in the right panel - lies within the uncertainty of the eight runs. The scenario hit point (all-run average) which is more to the back of the ship is represented by a white dashed vertical line and a standard deviation given as two red short vertical lines.
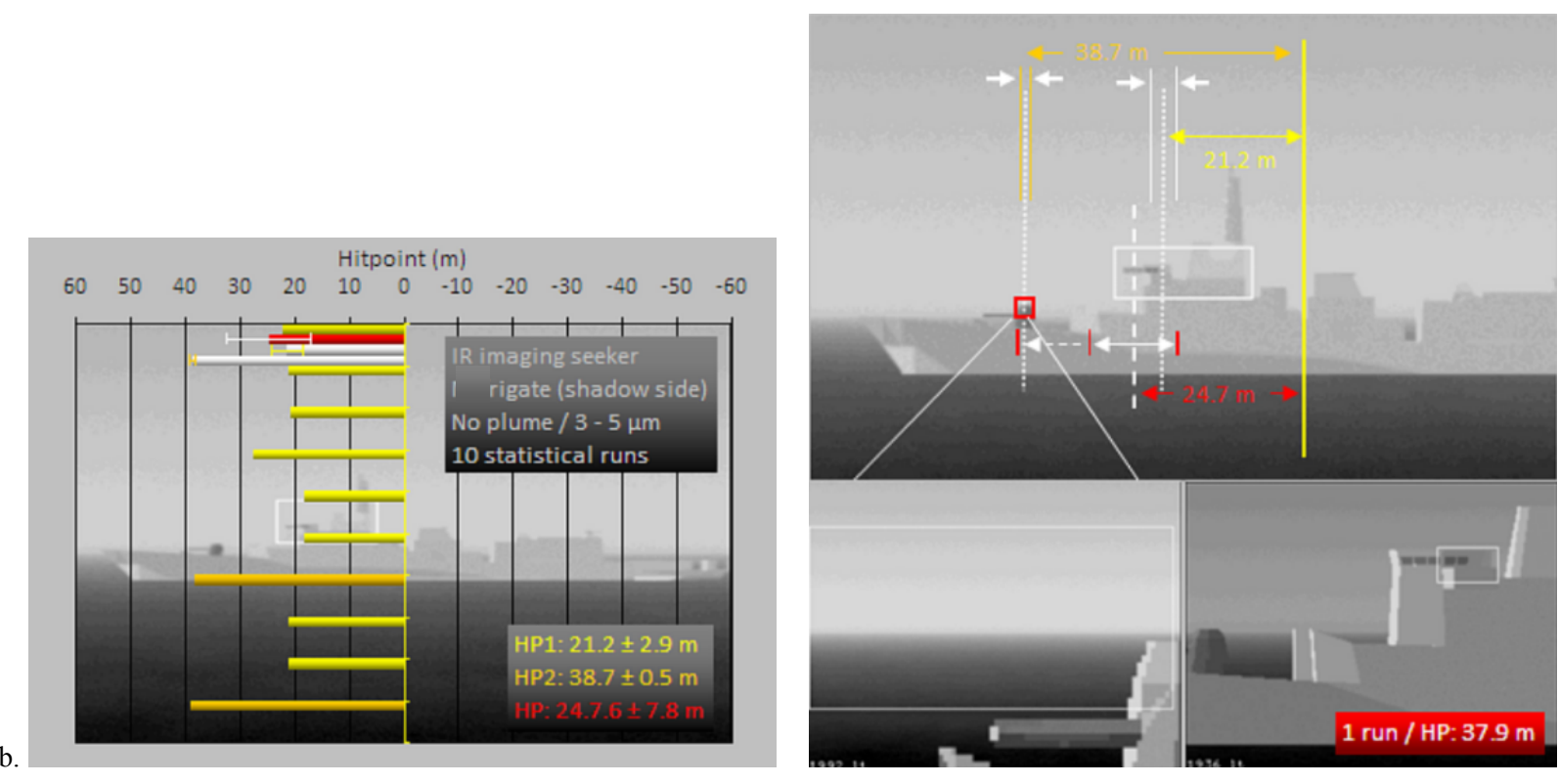

Figure $7 \mathrm{~b}$. Shown are two hit point preferences, marked by a different colour, for scenario 2 . Two runs have a preference to hit the ship way up front (approx. $39 \mathrm{~m}$ from the centre). The other eight runs converge at approx. $21 \mathrm{~m}$ from the centre in the bridge area (with a small standard deviation). The red bar designates the overall average and standard deviation. Right hand panel: a single run hit point, showing a preference for one of the two hit point areas (see above). The preferred target seems to be the front cannon. Note the two zoom views: earlier in the flight the missile aims at the bridge (right zoom) while it hits the front side of the cannon in the end stage (left zoom). 

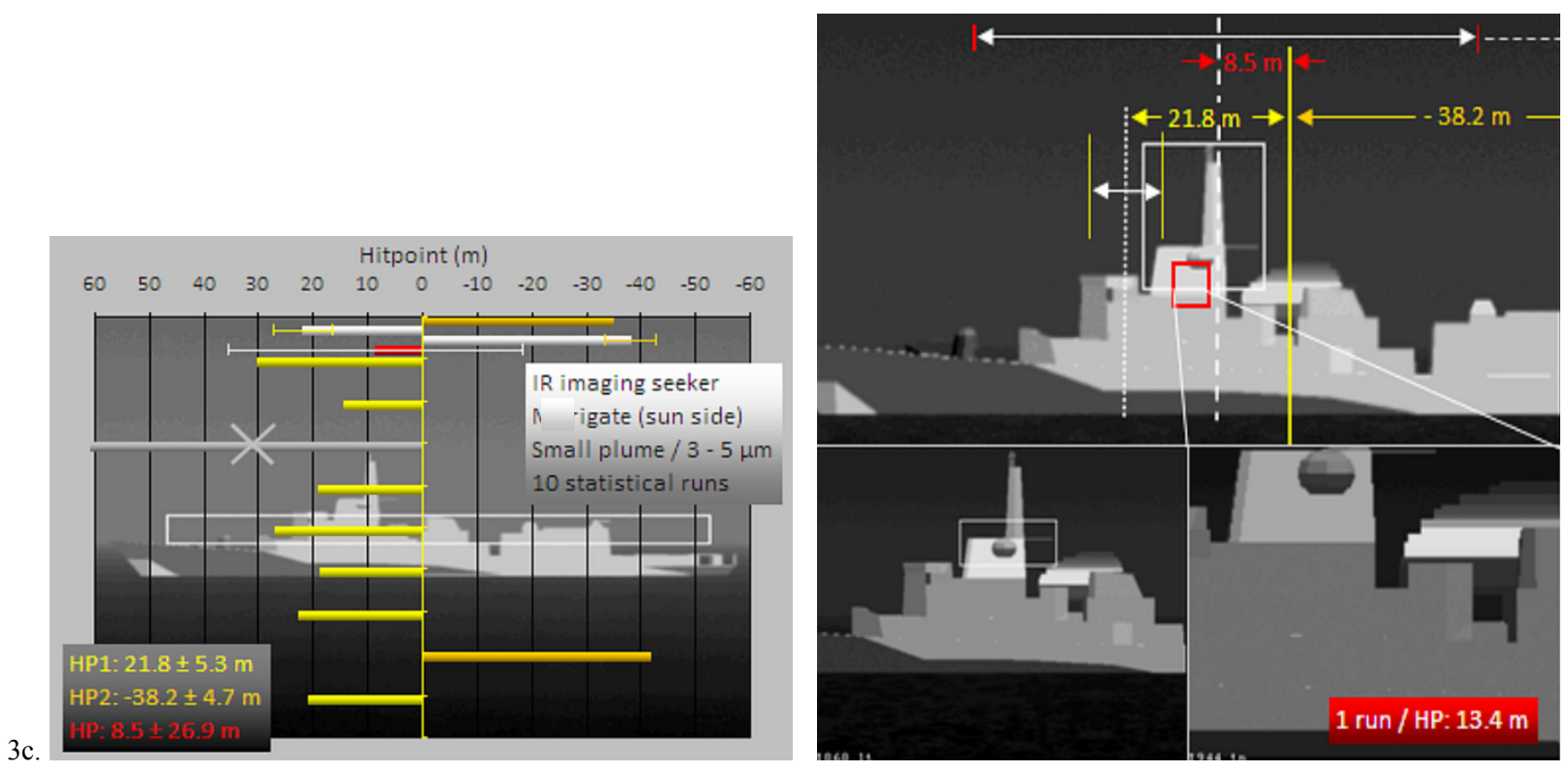

Figure 7c. Two preferred hit point areas are identified for scenario 3. One in the aft section of the ship and one in the bridge section. One run is assumed invalid. Note that the background picture in this top panel is for illustration only and does not include a plume. The size of the plume in the right hand panel can be clearly seen. Right hand panel: a single run hit point lies just behind the bridge in the mast section. The average of all nine successful runs is at $8.5 \mathrm{~m}$ from the centre of the ship. The standard deviation for all measurements is large $(26.5 \mathrm{~m})$ (due to the hit point aft section at $-38.2 \mathrm{~m}$ just outside the picture frame).
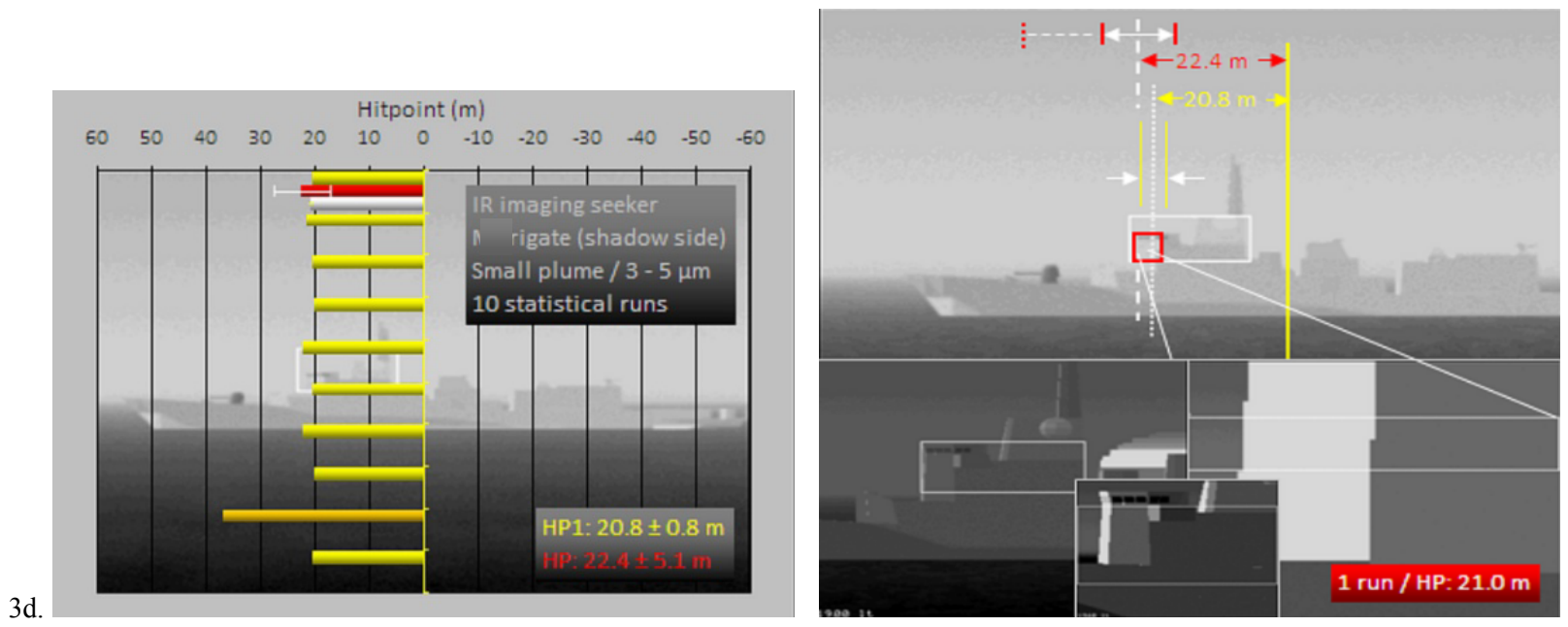

Figure 7d. Hit point results for scenario 4 showing a clear preference for the bridge section of the frigate, despite the plume present. Right hand panel: single run hit point result for which it is clear that the hit point is caused by the bright lit forward section of the bridge (see frame inset for details). 


\section{SUMMARY AND CONCLUSIVE REMARKS}

In this paper the tools for hit point analysis and countermeasure studies have been demonstrated for a selected number of scenarios.

The main conclusion for the hit point analysis is that no single aim point can be declared which is consistently the same for all ship signature conditions and for all exhaust plume conditions. For subsets of scenarios with similar signature conditions or similar plume conditions the hit points can be clustered in certain preferred areas. Depending on the scenario these preferred areas can cluster for example around the plume, the bridge etc. Even when the aim point within a certain scenario seems to be very consistent, it has to be noted that this is related to a very specific detail in the ship superstructure often only visible for a limited range of aspect angles, or occurring only for very specific atmospheric conditions. So no conclusions can be drawn form these simulations for improving the design of the ship.

For a full hit point analysis more scenarios should be used. Full analysis requires a full matrix with, for instance, attack direction, wind speed, and ship speed. To increase the selection of seekers in the EWM environment, implementation of the correlation tracker and the centroid tracker as used in the seeker models 1 and 2 could be considered. Also the tuning of the seeker parameters for the improved image quality should be part of a further study. Even more important, the implementation of a specific tracking algorithm for the final homing section, including the decision logic for the transition from midcourse guidance to the final homing guidance will require a significant effort.

The results presented here and for the other scenarios have been analysed manually. For more extensive studies with multiple runs over multiple aspect angles for multiple scenarios it will become useful to develop tools to set up the hit point simulations automatically. Also tools for automatic analysis of the results could be useful. Another issue is the run time for the simulations. Especially for the imaging seekers it could be useful to try to reduce the run time of the simulations.

The countermeasure effectiveness studies discussed in this paper, indicate that the success of a decoy deployment depends more on the capability of launching the decoy into the correct position with respect to the line of sight of the missile seeker to the target ship than on the detailed infrared signature characteristics of the decoys and the ship as long as the ship signature remains below the decoy intensity. This combined with the level of detail the seeker can see in the scene at the range where the decision whether to track the ship or the decoy has to be taken, justifies the use of much less detailed representation of ship and decoys used in the simulation. However, it is expected though that the EOSTAR image sequences for ships and decoy screens, which are currently being implemented for newly built vessels, result in a more realistic and certainly synergistic system of models.

In conclusion: a powerful toolset to assess the survivability of ships against infrared guided missiles has been set up by linking the three TNO model suites EOSTAR, EOSM, and EWM. The application of the toolset for hit point analysis and for countermeasure effectiveness has been demonstrated.

\section{ACKNOWLEDGEMENT}

The author would like to thank the Royal Netherlands Navy and the Defence Materiel Organisation for supporting this work. Special thanks go to Aad de Jong of the Ministry of Defence for stimulating discussions.

The author also acknowledges the contributions of the colleagues at TNO, in particular Wim de Jong, Robin Schoemaker and Ronald van Sweeden and the EOSTAR team.

\section{REFERENCES}

1. H.M.A. Schleijpen, "Evaluation of infrared signature suppression of ships", in Targets and Backgrounds: Characterization and Representation II, W.R. Watkins, and D. Clement, editors, Proc. SPIE Vol. 2742, pages 245254, Orlando (FL), USA, Apr. 1996.

2. H.M.A. Schleijpen, F.P. Neele, "Ship Exhaust Gas Plume Cooling", SPIE-2004 Orlando Targets and Backgrounds X: Characterisation and Representation, SPIE Vol. 5431, p 66, 2004. 
3. F.P. Neele, and W. de Jong, "Prewetting systems as an IR signature control tool" in Targets and Backgrounds VIII: Characterization and Representation, W.R. Watkins, D. Clement, and W.R. Reynolds, editors, Proc. SPIE Vol. 4718, pages 156-163, Orlando (FL), USA, Apr. 2002

4. W. de Jong, S.P. van den Broek, and R. van der Nol, IR seeker simulator to evaluate IR decoy effectiveness in Targets and Backgrounds VIII: Characterization and Representation, W.R. Watkins, D. Clement, and W.R. Reynolds, editors, Proc. SPIE Vol. 4718, pages 164-172, Orlando (FL), USA, Apr. 2002

5. Wim de Jong, Frans A.M. Dam, Gerard J. Kunz, Ric. M.A. Schleijpen, "IR seeker simulator and IR scene generation to evaluate IR decoy Effectiveness", London 2004, Technologies for Optical Countermeasures, SPIE Vol 5614-11

6. Robin Schoemaker, Ric Schleijpen: "Evaluation Tools for the Effectiveness of Infrared Countermeasures and Signature Reduction for Ships", SPIE “infrared Imaging Systems: Design, Analysis, Modelling and Testing XXI, Vol 7662 paper 26.

7. F.A.M. Dam, W. de Jong, H.R. Mühren, "Naval ECM, a quantitative analysis", TNO Report TNO-DV 2008 A514, June 2009, Stg Confidentieel.

8. Marianne A. C. Degache, Alexander M. J. van Eijk, Dimitris Tsintikidis, Stephen M. Hammel, "EOSTAR Pro: a flexible extensive library to assess EO sensor performance" Optics in Atmospheric Propagation and Adaptive Systems, Toulouse 2010, SPIE Vol 7828-03.

9. A.L. Mieremet, W. de Jong, "EOSM validation using SAPPHIRE data", TNO report TNO-DV 2008 A253, June 2008, NATO restricted. 